\section{RESPONSE LETTER TO DRS. MARTIN AND ALESSI}

To the Editor: Drs. Martin and Alessi raise valid concerns about three Minimum Data Set (MDS) measures. Each concern was already discussed, at some length, in the original report. ${ }^{1}$ Strengths and limitations of MDS-based research derive in part from the simplicity of its measures. Debate about the role of the MDS in research is almost as old as the instrument itself ${ }^{2,3}$ but has not prevented many worthwhile studies from being performed. We welcome Martin and Alessi's new data but cannot agree with the conclusions they make, which seem to reflect some misconceptions about insomnia, its measurement, and consequences of imprecise measurement.

Insomnia is a patient complaint not generally defined by any criterion-standard objective measure. Insomnia is "a complaint of difficulty initiating sleep, difficulty maintaining sleep, or waking up too early or sleep that is chronically nonrestorative or poor in quality." 4 The complaint occurs despite adequate opportunity for sleep and leads to daytime impairment, as defined by any of nine symptoms. In their own study, Martin and Alessi instead defined insomnia according to observed daytime sleepiness, actigraph-estimated sleep time, and an arbitrarily assumed sleep opportunity of 10 hours (letter above) or 8 hours. ${ }^{5}$ This is particularly problematic, because many individuals who obtain little nocturnal sleep have no complaints about it, experience no daytime symptoms, or know that to alleviate such symptoms they only need to get more sleep. ${ }^{4}$ The MDS may well identify insomnia that is really a complaint and a problem more effectively than actigraphy can.

Actigraphy does not record any physiological parameter that defines sleep. ${ }^{6}$ Although agreement with laboratory-based polysomnography is excellent in normal subjects, performance is considerably worse in insomniacs, $, 7,8$ and its use in insomnia has not been fully validated. ${ }^{9,10}$ Substantial differences in numbers of insomniacs identified using the MDS, actigraphy, and the Pittsburgh Sleep Quality Index (PSQI) are not surprising. The PSQI was designed to assess sleep quality and was validated in good and poor sleepers, not strictly insomniacs. ${ }^{11}$ The instrument includes items about daytime sleepiness, enthusiasm, bad dreams, and use of hypnotics. It generates a continuous score likely to show a unimodal distribution that would make any dichotomized classification highly sensitive to the chosen cutoff. ${ }^{11}$

Martin and Alessi confirmed that each of their own MDS-identified hypnotic users used hypnotics but found that the MDS missed several actual hypnotic users. However, their subjects were selected for hypersomnolence and may have used hypnotics with high frequency, because sleepiness is one of the main side effects. Five of the 6 months for which our study enumerated falls were assessed using the MDS item that showed moderate rather than fair agreement with chart abstraction. ${ }^{12}$ Even if all the new data cited by Martin and Alessi are interpreted as they do, the risk raised by some missed cases of insomnia, hypnotic use, and falls is most likely a conservative bias against detection of the reported associations. Had it been possible to identify missed cases of insomnia, hypnotic use, or falls, our conclusions would have been substantially different only if missed cases showed systematically different relationships between the variables under study. Martin and Alessi offer no specific reason to suspect that this occurred.

A widely generalizable, longitudinal investigation of 34,163 subjects (more than $94 \%$ of those targeted for inclusion) and many potential confounds could not be performed with actigraphy, thorough chart review, or polysomnography. As noted in the report, the conclusions should not be overinterpreted to justify hypnotic use in nursing homes unless the findings are confirmed in future clinical trials. In addition, investigators whose own research efforts are predicated on the ineffectiveness and dangers of hypnotics in older people should not exaggerate the findings. ${ }^{5}$ For example, we did not "conclude that ... reduction of insomnia using ... hypnotics may actually reduce the risk for subsequent falls,"13 although we did - and still do-note that the data "could support (this) speculation."

$$
\begin{array}{r}
\text { Alon Y. Avidan, MD, MPH } \\
\text { Sleep Disorders Center } \\
\text { Department of Neurology } \\
\text { University of Michigan Health System } \\
\text { Ann Arbor, MI }
\end{array}
$$

Brant E. Fries, $P h D$

Department of Health Management and Policy

School of Public Health

Institute of Gerontology

University of Michigan Medical School

Ann Arbor, MI

Kristina L. Szafara, PhD

Institute of Gerontology

University of Michigan Medical School

Ann Arbor, MI

Ronald D. Chervin, MD, MS

Sleep Disorders Center

Department of Neurology

University of Michigan Health System

Ann Arbor, MI

\section{ACKNOWLEDGMENTS}

Financial Disclosure: Alon Y. Avidan has served as consultant and is on the speakers forum for Takeda Inc., Sepracor, and Glaxo-SmithKlein and has served as a consultant for Neurocrine. This study was supported in part by the National Institute on Aging through Older Americans Independence Grant P60 A608808-10 and Training Grant T32 AG00114.

Author Contributions: Alon Y. Avidan: interpretation of data and preparation of manuscript. Brant E. Fries: interpretation of data, preparation of manuscript, acquisition of subjects, and data analysis and design. Kristina L. Szafara and Ronald Chervin: interpretation of data, preparation of manuscript, and data analysis and design.

Sponsor's Role: None.

\section{REFERENCES}

1. Avidan AY, Fries BE, James ML et al. Insomnia and hypnotic use, recorded in the Minimum Data Set, as predictors of falls and hip fractures in Michigan nursing homes. J Am Geriatr Soc 2005;53:955-962. 
2. Teresi JA, Holmes D. Should MDS data be used for research? Gerontologist 1992;32:148-149.

3. Hawes C, Phillips CD, Mor V et al. MDS data should be used for research. Gerontologist 1992;32:563-564.

4. The International Classification of Sleep Disorders. Westchester, IL: American Academy of Sleep Medicine, 2005.

5. Alessi CA, Martin JL, Webber AP et al. Randomized, controlled trial of a nonpharmacological intervention to improve abnormal sleep/wake patterns in nursing home residents. J Am Geriatr Soc 2005;53:803-810.

6. Rechtschaffen A, Kales A, eds. A Manual of Standardized Terminology, Techniques, and Scoring System for Sleep Stages of Human Subjects. Washington, DC: U.S. Department of Health, Education, and Welfare, Public Health Service, NIH/NIND, 1968.

7. Chambers MJ. Actigraphy and insomnia: A closer look. Part 1. Sleep 1994;17: 405-408, discussion 408-410.

8. Hauri PJ, Wisbey J. Wrist actigraphy in insomnia. Sleep 1992;15:293-301.

9. National Institutes of Health State of the Science Conference Statement on Manifestations and Management of Chronic Insomnia in Adults, June 13-15, 2005. Sleep 2005;28:1049-1057.

10. Chesson A Jr, Hartse K, Anderson WM et al. Practice parameters for the evaluation of chronic insomnia. An American Academy of Sleep Medicine report. Standards of Practice Committee of the American Academy of Sleep Medicine. Sleep 2000;23:237-241.

11. Buysse DJ, Reynolds CF 3rd, Monk TH et al. The Pittsburgh Sleep Quality Index. A new instrument for psychiatric practice and research. Psychiatry Res 1989;28:193-213.

12. Hill-Westmoreland EE, Gruber-Baldini Falls documentation in nursing homes. Agreement between the Minimum Data Set and chart abstractions of medical and nursing documentation. J Am Geriatr Soc 2005;53:268-273.

13. Martin JL, Alessi CA. Limited validity of Minimum Data Set items on sleep and hypnotic use in predicting falls and hip fracture in nursing home residents. J Am Geriatr Soc 2006;54:1150-1151.

\section{MORGAGNI'S HERNIA IN AN OLDER ADULT WOMAN WITH A SUBPHRENIC ABSCESS}

To the Editor: A 74-year-old Haitian woman with prior history of recent stroke, untreated hypertension, and chronic schizophrenia was transferred to the geriatric inpatient service with a history of worsening abdominal pain after treatment for urinary tract infection. The abdominal pain had been associated with severe vomiting. Abdominal examination revealed decreased bowel sounds, moderate distention, and mild tenderness to palpation, without signs of peritonitis. Initial laboratory tests showed an elevated white blood cell count with left shift and dehydration. Intravenous piperacillin/tazobactam and metronidazole were continued for a suspected liver abscess previously seen on ultrasonic imaging. The gastroenterology service was consulted, and esophagogastroduodenoscopy showed distal esophagitis and a hiatal hernia. An abdominal computed tomography $(\mathrm{CT})$ with contrast revealed a $10-\mathrm{cm}$ right liver lobe cyst in addition to a questionable subphrenic abscess. A small bowel obstruction was also identified.

The differential diagnoses for this patient at the time included pyogenic abscess, hydatid cyst, amebic abscess, and malignancy. Bacterial cultures and hydatid titers were negative. Amebic titers were equivocal, and tumor markers were within normal limits. The surgery service was consulted, but the recent stroke precluded open drainage. CT-guided percutaneous drainage of the abscess was performed. Fluid analysis was negative for bacteria, syphilis, tuberculosis, and malignancy. Cytology showed fungal species, and antifungal treatment was added. Three weeks after the initial onset of symptoms, a chest CT revealed new bilateral pleural effusions. The patient began to deteriorate, and emergent surgical intervention revealed a right anterior diaphragmatic hernia with strangulated necrotic small bowel, which resulted in a right subphrenic abscess due to the bowel perforation. Small bowel resection was done. The patient recovered and was transferred to a skilled nursing facility.

Morgagni hernias are rare, and delayed diagnosis is common. In adults they can be asymptomatic or present as an incidental finding on chest radiography. Little is known about these types of hernias, because they account for only $2.5 \%$ to $5 \%$ of all surgically treated diaphragmatic hernias. They most likely are caused by a congenital defect in the fusion of the septum transversus of the diaphragm with the costal arches. ${ }^{1}$ Intraperitoneal pressure gradually stretches the diaphragmatic defect, giving rise to the hernia. Although the potential space for Morgagni hernias is congenital, other factors such as rapid changes in abdominal pressure and degenerative changes in the aging diaphragm (presbydiaphragm) also contribute.

Symptoms, if present, consist of abdominal pain, nausea, vomiting, intestinal obstruction, shortness of breath, and chest tightness. ${ }^{2}$ Possible causes of sudden herniation include trauma, straining, lifting heavy weights, or intraabdominal pathology such as cancer or pancreatitis. ${ }^{1}$ In the described case, the likely precipitating factor was the increased abdominal pressure created by the patient's vomiting associated with her urinary tract infection.

A leak of the perforated bowel under the liver, producing a subphrenic abscess, complicated this patient's diaphragmatic hernia. The abscess made reaching the correct diagnosis difficult, because radiographically it was challenging to determine the exact location and connection with the small bowel. The need to consider an expanded differential diagnosis, including tropical parasites, for this patient from a developing country also delayed the final diagnosis.

Although initial symptoms can include respiratory distress, in this case, the pleural effusions and respiratory symptoms developed late in the patient's course. Respiratory symptoms are often a helpful aid, because they trigger the chest radiographs by which the diagnosis of Morgagni hernia is usually made. ${ }^{1,3}$ Recent recommendations include the use of CT scans or magnetic resonance imaging to differentiate between Morgagni hernias and other mediastinal fat-containing masses. ${ }^{4}$

Anterior diaphragmatic hernias are challenging to diagnose, especially in elderly patients who present with atypical symptoms such as those seen in this patient. In this case, the diaphragmatic hernia and its contents were not evident on imaging and not confirmed until surgical intervention. If found in older adults, Morgagni hernias should be carefully considered in the differential diagnosis of abdominal pain and respiratory distress before being dismissed as long standing and clinically insignificant.

S. Liliana Oakes, $M D$ David V. Espino, $M D$ Nancy Santana, $M D$ Gerardo E. Carcamo, MD Department of Family and Community Medicine University of Texas Health Science Center at San Antonio NIX Hospital San Antonio, TX 